\title{
Thrombospondin type-1 domain-containing 7A -a new player in membranous nephropathy
}

n 2009, the podocyte receptor phospholipase A2 receptor 1 (PLA2R1) was identified as the first major autoantigen involved in the pathogenesis of adult idiopathic membranous nephropathy (iMN). Subsequent studies confirmed circulating autoantibodies to PLA2R1 in approximately $70 \%$ of affected patients; however, the cause of disease in the remaining $30 \%$ of patients was unknown. New findings, published in The New England Journal of Medicine identifies thrombospondin type-1 domain-containing 7A (THSD7A) as a novel membranous nephropathy-causing antigen, estimated to underlie 5-10\% of cases of $i \mathrm{MN}$ in patients who are seronegative for anti-PLA2R1 antibodies. "This finding furthers our understanding of the pathophysiological basis of iMN," says researcher Gerard Lambeau. "It also allows the identification and monitoring of patients positive for anti-THSD7A, as is already done using commercially available blood tests for patients who are positive for anti-PLA2R1."

IMN is an autoimmune disease that commonly leads to nephrotic syndrome in adults. The $30 \%$ of patients with iMN who do not have circulating autoantibodies to PLA2R1 are thought to possess other endogenous glomerular antigens. To identify circulating antibodies against novel glomerular antigens, Lambeau and colleagues screened sera from patients with iMN without anti-PLA2R1 antibodies, those with anti-PLA2R1 antibodies, those with other glomerular diseases, and healthy controls against a total protein extract from human glomeruli and against the three paralogues of human PLA2R1.

"As we previously found that the anti-PLA2R1 autoantibodies are conformational and recognize PLA2R1 only as a folded antigen, we performed the screen using nonreducing conditions," explains Lambeau.
Seven of 152 serum samples from patients with membranous nephropathy showed reactivity to a glomerular protein that was approximately $250 \mathrm{kD}$ in size. All seven samples were from patients who were seronegative for anti-PLA2R1 antibodies; samples from healthy controls, patients with other glomerular diseases or patients who were seropositive for antiPLA2R1, did not react. Analysis of 365 samples from an independent cohort from Boston University identified 10 additional patients who were seronegative for antiPLA2R1 but reactive to the putative $250 \mathrm{kD}$ autoantigen. Mass spectometry of digested peptides identified this protein as THSD7A. All 17 reactive serum samples -but not samples that were positive for anti-PLA2R1 or control samples-were able to immunoprecipitate THSD7A from glomerular lysates.

The researchers performed immunofluorescence staining of renal biopsy samples to assess THSD7A expression. THSD7A colocalized with nephrin on or near podocyte foot processes. Staining for THSD7A was enhanced in biopsy samples from patients who were positive for anti-THSD7A antibodies compared with samples from patients who had anti-PLA2R1 serum antibodies. Like PLA2R1, IgG4 was identified as the predominant IgG subclass of antiTHSD7A, and immunohistochemistry revealed colocalization of IgG4 and THSD7A in biopsy samples from THSD7A-seropositive patients.

"We therefore conclude that patients with iMN can be classified into three different subgroups: those positive for anti-PLA2R1 autoantibodies but negative for anti-THSD7A and representing the major subgroup with about $70 \%$ of cases; those positive for anti-THSD7A but negative for anti-PLA2R1 and representing $5-10 \%$ of patients; and finally those negative for both autoantibodies

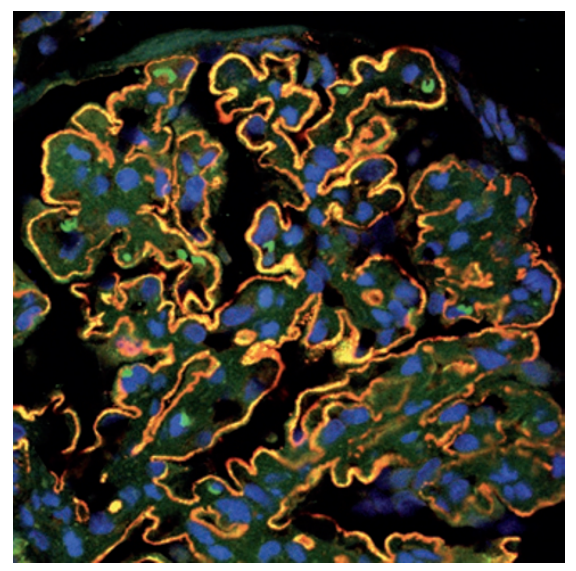

Colocalization of IgG4 and THSD7A in the peripheral capillary wall and the glomerular basement membrane of a kidney biopsy sample of a patient with idiopathic membranous nephropathy and positive for anti-THSD7A. Image courtesy of U. Helmchen, Hamburg, Germany.

and representing another subgroup of about $20 \%$ of cases, suggesting the possible existence of other unknown antigen(s)," says Lambeau.

The researchers state that novel strategies should now be designed to screen sera from patients with iMN who are negative for both PLA2R1 and THSD7A autoantigens to identify putative novel antigens. "Future research should also be directed towards elucidating the normal function of PLA2R1 and THSD7A in podocytes to help us better understand the molecular mechanisms of pathogenesis of iMN" explains Lambeau. "Our discovery represents an excellent example of international collaboration between teams from France, Germany and the USA, with the decision to combine our independent work and accelerate research towards a better understanding of a rare but serious autoimmune kidney disease that affects thousands of people worldwide."

Susan J. Allison

Original article Tomas, N. M. et al. Thrombospondin type-1 domain-containing 7A in idiopathic membranous nephropathy. N. Engl. J. Med. doi:10.1056/NEJMoa1409354 